\title{
Evaluation of micro structure by fusion of three dimensional shape measurement and ultimate analysis using SEM
}

\author{
Yasuhiko Arai \\ Kansai University, Department of Mechanical Engineering,3-3-35 Yamate-cho, Suita, Osaka, Japan
}

\begin{abstract}
The 3-D measurement method by SEM has already been proposed by using the principle of projection moiré. In this method, by the mechanism of producing some shadows of grid on the surface of the object by back scattering electron beam, a micro-size object can be measured in high resolution. However, in the case of measurement of industrial elements, not only 3-D shape measurement, but also the physical properties of material is sometimes required to check strongly for practical uses. For example, the precise patterning lines and the existence of some kinds of atoms in IC circuits are parameters concerning important feature of the circuits. The distribution of atoms in fractured sections is also an important feature in destructive inspection. In this paper, the realization of such inspection technology by combining the ultimate analysis and 3-D measurement method by SEM is discussed. The new measurement technology by fusion of 3-D shape measurement and ultimate analysis is proposed. In experimental results, the 3-D shape measurement of the micro object made of alloy material is measured. From measured results, the validity of the new method is discussed.
\end{abstract}

\section{Introduction}

Optical measurement technology is one of the important measurement methods in the industries. In 1980s, fringe scanning technologies brought a success concerning high resolution power in the optical measurement methods. Now, many small elements are getting to be used in the industry under the stream of nanotechnology.

However, general optical measurement methods have a problem with the diffraction phenomenon. That is, lateral resolution power of optical methods is not high. The smaller the measured objects are, the larger the influence of this problem in the measurement is. To solve this problem, the new measurement method based on the optical measurement technology using SEM (Scanning Electron Microscope) was proposed by using electron instead of photons. Then, a high precision measurement is realized by this technology.

However, when the small elements are employed in the industries, not only the 3-D shape, but also the purity of materials from the elements is strongly required for getting a high performance. Therefore, in the measurement of micro elements, both the 3-D shape measurement and the ultimate analysis of the element would be required strongly.

In this paper, a new measurement method that simultaneously realizes the new high resolution 3-D measurement method using SEM and the ultimate analysis is proposed. In the experimental results, the validity of the method is confirmed.

\section{Principle of measurement}

\subsection{Three dimensional shape measurement}

In the optical system in the chamber of SEM, the coordinate system shown in Fig. 1 is assumed. Here, assuming that the positions of the gun and the detector are known, the coordinate of the measured point can be calculated.

In 3-D shape measurement, the position of the measured point $\mathrm{P}_{0}$ on SEM image is detected by defining the plane1 which includes the point $-\mathrm{P}_{0}$, the position of gun, and the position of the detector. That is, the electron, which is emitted from the gun, changes to a back scattering electron at the point $\mathrm{P}_{1}$ on the measured object. And, the back scattering electron reaches to the detector through a space of the grating on the plane-3. Then, the coordinate of the measured point $\mathrm{P}_{1}$ on the object corresponds to the coordinate of the $\mathrm{P}_{0}$ on the bottom plane- 0 according to the coordinate system of the SEM.

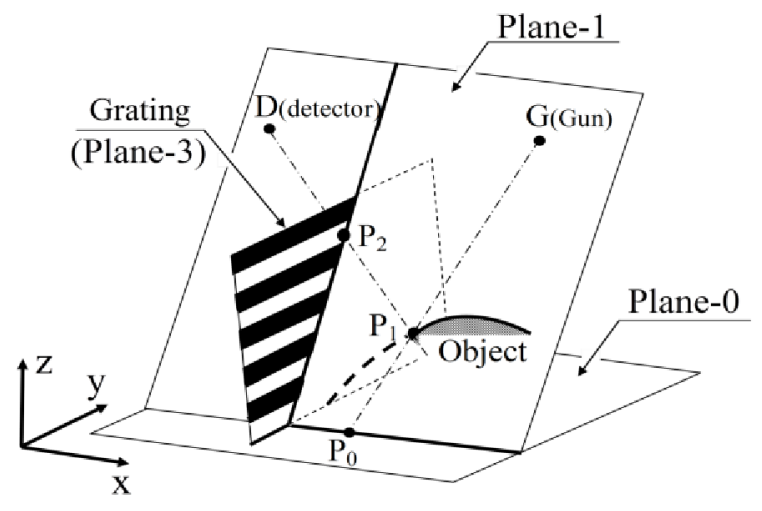

Figure 1. Coordinate system for analysis

$\overline{\text { Yasuhiko Arai: arai@kansai-u.ac.jp }}$

This is an Open Access article distributed under the terms of the Creative Commons Attribution License 4.0, which permits unrestricted use, distribution, and reproduction in any medium, provided the original work is properly cited. 
Thus, the plane- 1 can be defined by three points of the coordinate system of SEM. That is, Plane-1 can be defined by using the point-G (the gun), the point-D (the detector), and point- $\mathrm{P}_{0}$. Then, the straight line $\mathrm{G}-\mathrm{P}_{0}$ can be defined on the plane- 1 .

If the phase of the fringe at point- $\mathrm{P}_{0}$ is detected precisely, the point that has the same phase value as the phase of the fringe at point $-\mathrm{P}_{0}$ which is detected as the SEM image can be defined as the point $\mathrm{P}_{2}$ on the plane-3. Of course, the position of the plane- 3 (the grating) is known. And, the point $\mathrm{P}_{2}$ exists on the plane-1. Then, the straight line $\mathrm{D}-\mathrm{P}_{2}$ can be also defined on the plane- 1 . Consequently, it can be confirmed that the cross point of the straight line D- $\mathrm{P}_{2}$ and the straight line $\mathrm{G}-\mathrm{P}_{0}$ on the plane-1 is namely the measured point $\mathrm{P}_{1}$. Under this process, the coordinate of all measured points can be detected.

\subsection{Ultimate analysis}

In this study, X-ray micro analysis system (EMAX Evolution/EMAX ENERGY produced by Horiba) is employed as an ultimate analysis system. The detecting principle of ultimate analysis can be explained as follows: In this system, the electron that is emitted to an atom by a gun of SEM in a high speed makes characteristic X-rays of which an energy corresponds to the difference energy between electrons of outside and inside nuclear shells. Then, the atom can be defined by the quantity of the energy of the characteristic X-rays in this system.

\section{Experiment result and discussion}

\subsection{Optical system using SEM}

The new optical system using SEM (S-4300 produced by Hitachi) and X-ray micro analysis system is shown in Fig.2. The new optical system using electrons is constructed in the chamber of the SEM as shown in Fig.3. The optical system uses the grating of which the pitch is $40 \mu \mathrm{m}$. The grating shown in Fig.3(a) is produced of Siwafer by the silicon fabrication.

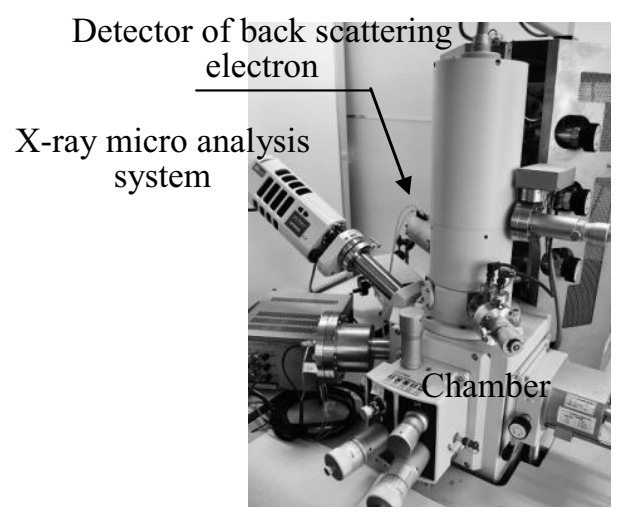

Figure 2. Experimental apparatus

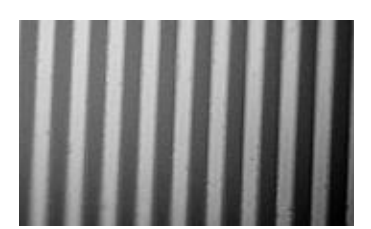

(a) Grating (pitch=40 $\mu \mathrm{m})$

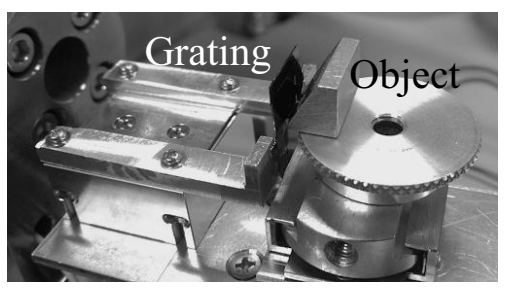

(b) Measured object and grating

Figure 3. Measured object and grating in the chamber

\subsection{Ultimate analysis}

The section of the object, which is bundled up some fine wires made of aluminium, silver, copper, and, tin, is observed by this system. The result is shown in Fig.4.

\subsection{Technology for fusion of three dimensional shape measurement and ultimate analysis}

3-D shape measurement and the ultimate analysis of a plate made of alloy (brass) are performed. In Fig.5(a), the fringe image for 3-D shape measurement by the measurement system is shown. The fringes are produced by electrons. The region surrounding by white broken line is the test area in this experiment. This area is available area after processing of the Hanning window. The 3-D shape from the fringe image is calculated by using the spatial fringe analysis method. The result is shown in Fig.5(b).

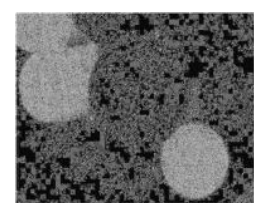

(b) $\mathrm{Al}$

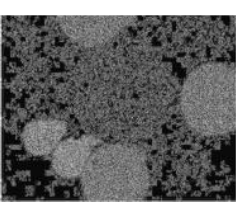

(c) Ag

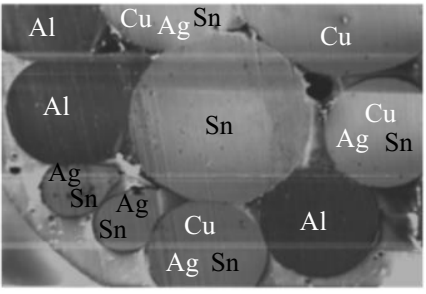

(a) Many kinds of material

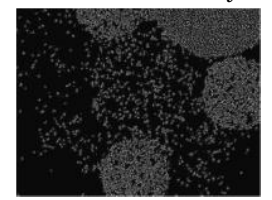

(d) $\mathrm{Cu}$

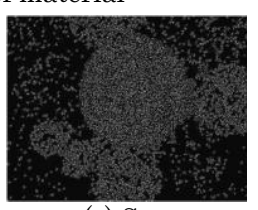

(e) $\mathrm{Sn}$
Figure 4. Ultimate analysis 


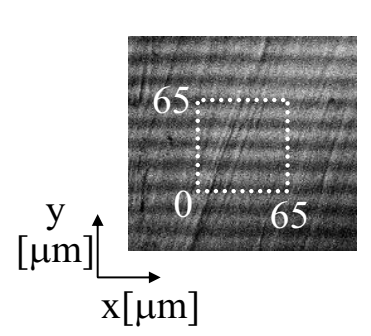

(a)Fringe image

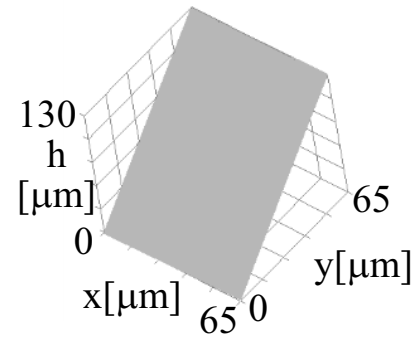

(b)3-D measurement
Figure 5. Fringes and 3-D shape measurement result

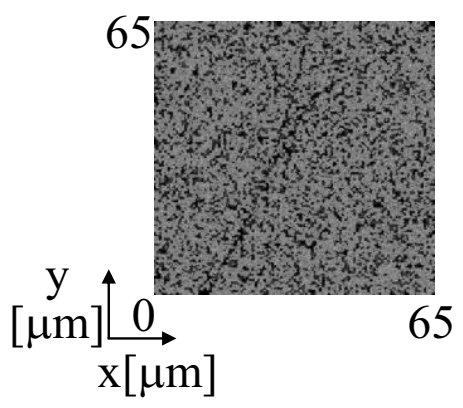

(a) Copper

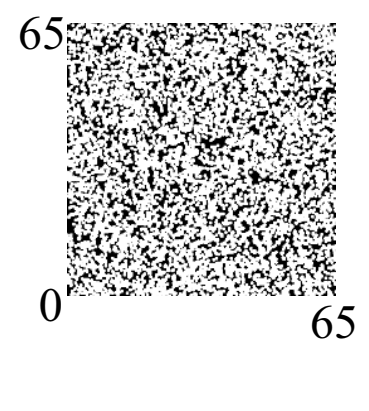

(b) Zinc
Figure 6. The copper and the zinc distributions

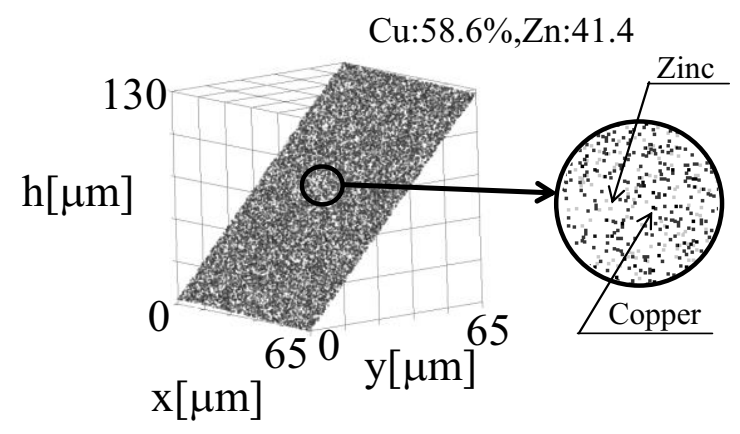

Figure 7. 3-D shape measurement with ultimate analysis result

And, the results of ultimate analysis for the copper and the zinc distributions are also shown in Fig.6 (a) and (b). In these results, it can be confirmed that the content of $\mathrm{Cu}$ is $58.6 \%$, and $\mathrm{Zn}$ is $41.4 \%$.

The measured result of 3-D shape measurement that is superimposed with the ultimate analysis result is shown in Fig.7. In the practical result, each element $(\mathrm{Cu}$ and $\mathrm{Zn})$ is shown by color image.
From this result, it can be confirmed that the fusion of 3-D shape measurement and ultimate analysis can be performed in one image.

\section{Conclusion}

In this paper, the fusing technology of a novel 3-D measurement method for micro size products and ultimate analysis is proposed by using the electron-beam of SEM. A new measurement method based on the principle of projection moiré is constructed. The ultimate analysis is performed by X-ray micro analysis. The threedimensional measurement and the ultimate analysis were simultaneously performed. These results are shown as one sheet of data.

In this measurement system, the grating which was produced of a silicon-wafer under the silicon-process based on the semiconductor fabrication technology was used as a physical rigid grid. Fringe image as shadows of the grating was analyzed by the spatial fringe analysis method. A flat plate that is made of the alloy (brass) was measured in order to confirm the validity of the measurement principle of this new measurement system. It is confirmed that the very high resolution threedimensional measurement can be performed by the new method. In the close future, IC-circuits produced by $\mathrm{Si}$ and the fractured sections will be evaluated by this method.

\section{References}

1. G. Binning, H. Rohrer, Helv. Phys. Acta, 55, 726 (1982).

2. D. Malacara, Optical shop testing, 2ed (John Wiley \& Sons, New York, 1992).

3. J. I. Goldstein, Scanning Electron Microscopy and $X$ Ray Microanalysis ( Plenum press, New York, 1981).

4. L. Reimer, Transmission Electron Microscopy (Springer-Verlag, Berlin Heidelberg, 1984).

5. T. Suganuma, J. Electron Microscopy , 34, 328 (1985).

6. J. W. Dally, D. T. Read, Experimental Mechanics, 33, 270 (1993).

7. D. M. Meadows, Appl. Opt., 9, 942 (1970).

8. H. Takasaki, Appl. Opt., 9, 1467 (1970).

9. Y. Arai, S. Yokozeki, J. Modern Optics, 53, 2641 (2006).

10. D. Post, B. Han, P. Ifju, High Sensitivity Moiré (Springer-Verlag, New York, 1994).

11. Y. Arai, S. Yokozeki, T. Yamada, Appl. Opt., 34, 4877 (1995).

12. Y. Arai, S.Yokozeki, Appl. Opt., 38, 3503 (1999).

13. Y. Arai, S.Yokozeki, Int. J. Japan Soc. Prec. Eng., 31, 301(1997).

14. Y. Arai, J. Metrology society of India, 26, 69(2011). 